\title{
Respiratory Properties of Mitochondria Isolated from Conidia of Neurospora crassa
}

\author{
By RUBEN ORTEGA PEREZ,* \\ MEHRBANOU MICHÉA-HAMZEHPOUR AND GILBERT TURIAN \\ Laboratoire de Microbiologie générale, Département de Biologie végétale, Université de \\ Genève, $\mathrm{CH}-1211$ Genève 4, Switzerland
}

(Received 26 March 1981; revised 3 June 1981)

Mitochondria from conidia of Neurospora crassa were isolated and purified by a simple and rapid technique. Electron micrographic analysis and isopycnic centrifugation on a sucrose density gradient revealed a heterogeneous population.

Succinate, citrate, 2-oxoglutarate, NADH and ascorbate + tetramethyl-p-phenylenediamine (TMPD) were oxidized by the mitochondria. Oxygen consumption was totally inhibited by antimycin $A$ or cyanide. The electron transport chain was coupled to sites 1 and 2 of phosphorylation. Oligomycin, atractyloside and 2,4-dinitrophenol prevented energy coupling.

\section{INTRODUCTION}

Respiratory properties of mitochondria isolated from mycelia at different growth stages of Neurospora crassa wild type are now well characterized (Hall \& Greenawalt, 1967; Weiss et al., 1970; Lambowitz et al., 1972a, $b$; von Jagow et al., 1973).

It has been reported that conidial mitochondria of $N$. crassa have a low rate of oxygen consumption and that this respiration is predominantly cyanide insensitive (Colvin et al., 1973) and fails to perform oxidative phosphorylation (Greenawalt et al., 1972). However, in a preliminary study, Hall \& Greenawalt (1964) showed that mitochondria extracted from conidia of $N$. crassa were able to carry out oxidative phosphorylation. To clarify this discrepancy, we have developed an improved procedure for the extraction of conidial mitochondria of $N$. crassa (Ortega Perez et al., 1977) which enables us to isolate large quantities of relatively pure mitochondria which show structural integrity and well-preserved respiratory functions. After extraction under these improved conditions, conidial mitochondria possess a functional electron transport chain which is partially coupled to oxidative phosphorylation and totally sensitive to cyanide and antimycin A. Heterogeneity of mitochondria was observed by electron microscopy and isopycnic centrifugation.

\section{METHODS}

Organism and cultural conditions. The wild type of Neurospora crassa strain STA, 262A was obtained from the Fungal Genetic Stock Center, Humboldt State University, Arcata, California, U.S.A. Conidia were produced by inoculating 1.81 Fernbach flasks containing $200 \mathrm{ml}$ Neurospora Minimal Medium (Difco) and 2\% (w/v) agar with $2 \times 10^{8}$ conidia. Germination and growth proceeded for $2 \mathrm{~d}$ in the dark at $25^{\circ} \mathrm{C}$; the cultures were then kept for $3 \mathrm{~d}$ at the same temperature under continuous fluorescent light and aerated with humidified air.

Cell disruption and mitochondrial preparation. Conidia of $N$. crassa were harvested with distilled water, filtered through four layers of gauze and concentrated by centrifugation at $2500 \mathrm{~g}$ for $10 \mathrm{~min}$. The conidial pellet was further washed with distilled water and reconcentrated by low-speed centrifugation. Conidia were disrupted on a Vortex mixer modified as follows: the rubber ring was replaced by a disc of PVC ( $3 \mathrm{~mm}$ thick) with eight 
cylindrical tube-holders ( $25 \mathrm{~mm}$ height, $20 \mathrm{~mm}$ diam.). On an independent support, a perforated plate was fixed $95 \mathrm{~mm}$ above the disc, the holes coinciding with the tube-holders.

Each $1 \mathrm{~g}$ of conidia (wet wt) was suspended in $3 \mathrm{ml}$ extraction buffer $125 \mathrm{~mm}$-Tris/acetate, $0.44 \mathrm{M}$-sucrose, $0.7 \mathrm{~mm}$-EGTA, $10 \mathrm{~mm}$-dithiothreitol (DTT) and $0.3 \%(\mathrm{w} / \mathrm{v})$ bovine serum albumin, $\mathrm{pH} 7.21 ; 1 \mathrm{ml}$ of this suspension was then added to $2.5 \mathrm{~g}$ glass beads $(0.4$ to $0.5 \mathrm{~mm}$ diam.) in $25 \times 100 \mathrm{~mm}$ glass tubes. The tubes were then shaken in the modified Vortex mixer for $90 \mathrm{~s}$ at maximal speed and the homogenized cell suspensions were pooled. The beads were rinsed repeatedly with extraction buffer and added to the first suspension. The total volume of extraction buffer used per $\mathrm{g}$ conidia was $15 \mathrm{ml}$. Unbroken conidia and cellular debris were removed by centrifugation at $1500 \mathrm{~g}$ for $10 \mathrm{~min}$. The supernatant was then centrifuged at $12000 \mathrm{~g}$ for $15 \mathrm{~min}$ to yield the mitochondrial pellet, which was resuspended in $1 \mathrm{ml}$ extraction buffer (without EGTA and DTT) per g conidia and recentrifuged at $12000 \mathrm{~g}$ for $15 \mathrm{~min}$.

The final pellet of mitochondria was resuspended (to a final concentration of about $40 \mathrm{mg}$ protein $\mathrm{ml}^{-1}$ ) either, for measurements of respiration rates, in a medium that contained $25 \mathrm{~mm}$-Tris/acetate, $0.44 \mathrm{M}$-sucrose, $10 \mathrm{~mm}-\mathrm{KH}_{2} \mathrm{PO}_{4}, 5 \mathrm{~mm}-\mathrm{MgSO}_{4}$ and $0.2 \%(\mathrm{w} / \mathrm{v})$ bovine serum albumin $(\mathrm{pH} 7.2)$, or, for isopycnic centrifugation, in $50 \mathrm{~mm}$-Tris $/ \mathrm{HCl}, 1 \mathrm{~mm}$-EDTA and $35 \%(\mathrm{w} / \mathrm{v})$ sucrose $(\mathrm{pH} 7.2)$. All steps were performed at $4{ }^{\circ} \mathrm{C}$.

Isopycnic centrifugation. Portions of the mitochondrial suspension (15 to $20 \mathrm{mg}$ protein) were layered on a $20 \mathrm{ml}$ linear sucrose density gradient 135 to $65 \%(\mathrm{w} / \mathrm{v})$ sucrose in $50 \mathrm{~mm}$-Tris/ $\mathrm{HCl}, \mathrm{pH} 7.2$, containing $1 \mathrm{~mm}$-EDTA|. The tubes were centrifuged for $90 \mathrm{~min}$ at $129000 \mathrm{~g}$ (maximum speed) in an MSE Prepspin 50 centrifuge with a $3 \times 25$ swing-out rotor. Fractions $(0.8 \mathrm{ml})$ were removed from the bottom of the tubes by outflow with a peristaltic pump. The density of the fractions was calculated from the refractive index measured with an Erma refractometer. All steps were carried out at $4{ }^{\circ} \mathrm{C}$.

Respiratory measurements. Oxygen consumption was measured polarographically at $25^{\circ} \mathrm{C}$ with a Clark-type oxygen electrode (Gilson oxygraph model $\mathrm{k}-\mathrm{lC}$ ) on mitochondrial suspensions at a concentration of 0.4 to $0.8 \mathrm{mg}$ protein $(\mathrm{ml} \text { respiratory medium })^{-1}$. The final volume of the reaction chamber was $1.7 \mathrm{ml}$. Respiratory control. defined as state 3 (oxygen consumption in the presence of ADP)/state 4 (oxygen consumption when ADP was depleted), and ADP:O ratios were calculated by graphic analysis in response to the addition of ADP as described by Chance \& Williams (1955). All inhibitors except KCN were dissolved in dimethyl-sulphoxide (DMSO) and are indicated here at their final concentrations. DMSO alone had no effect on respiration.

The concentration of ADP in solution was determined by spectrophotometry at neutral pH $\left(\varepsilon_{260}=15.0 \mathrm{mmol}\right.$ $\left.1^{-1} \mathrm{~cm}^{-1}\right)$. Protein was determined by the Lowry method using bovine serum albumin as standard.

Electron microscopy. Portions of mitochondrial pellet were fixed at room temperature in $0.15 \mathrm{M}$-phosphatebuffered glutaraldehyde [final concentration $2.5 \%(\mathrm{w} / \mathrm{v})$ ] for $30 \mathrm{~min}$ and post-fixed in $2 \%(\mathrm{w} / \mathrm{v})$ osmium tetroxide for $1 \mathrm{~h}$ at the same temperature. Fixed mitochondria were treated with $2 \%(\mathrm{w} / \mathrm{v})$ uranyl acetate for $30 \mathrm{~min}$, dehydrated in ethanol and embedded in the medium described by Spurr (1969). Thin sections were stained with uranyl acetate and lead citrate. They were finally examined with a Zeiss M 10 electron microscope.

\section{RESULTS}

\section{Conidial disruption and mitochondrial isolation}

The concentration of conidia and the ratio of conidia to glass beads were critical for cell disruption. Most conidia (70 to $75 \%$ ) were broken after a treatment of $90 \mathrm{~s}$, whilst virtually complete breakage was obtained by treatment for $3 \mathrm{~min}$. However, the mitochondria extracted by the latter treatment had a reduced respiratory rate and had lost their capacity for oxidative phosphorylation. The average yield of mitochondrial proteins (11 determinations) was 6.0 (S.E.M. 0.2$) \mathrm{mg}$ (g conidial wet $\mathrm{wt})^{-1}$.

\section{Respiratory properties}

Table 1 summarizes the results obtained for oxygen consumption, respiratory control and ADP:O ratios of conidial mitochondria from $N$. crassa. The highest state 3 rates of respiration were obtained with exogenous NADH and succinate. The NAD ${ }^{+}$-linked substrates, citrate + malate and 2-oxoglutarate + malate, were also oxidized, but at a lower rate. The oxygen consumption with ascorbate alone was not significant but upon addition of tetramethyl-p-phenylenediamine (TMPD) it increased about 40 times. In the absence of mitochondria, the spontaneous oxidation of these substrates was nil.

The addition of ADP $(60$ or $120 \mu \mathrm{M})$ resulted in an increase of the respiratory rate with all substrates tested, except ascorbate + TMPD. The lowest respiratory control value was obtained with exogenous NADH while 2-oxoglutarate + malate showed the highest value. 
Table 1. Respiratory properties of mitochondria isolated from conidia of N. crassa

The respiratory activities were measured polarographically at $25^{\circ} \mathrm{C}$ as described in Methods. Substrate concentrations were: succinate, citrate, 2-oxoglutarate and ascorbate, $10 \mathrm{~mm}$; NADH, $2.5 \mathrm{~mm}$ : malate, $3 \mathrm{mM}$; and TMPD, $0.2 \mathrm{mM}$. The ADP concentration was $60 \mu \mathrm{M}$ for succinate and $120 \mu \mathrm{M}$ for the other substrates. Reactions were carried out with 0.4 to $0.8 \mathrm{mg}$ mitochondrial protein. Mean values \pm S.E.M. are given; the numbers of experiments done are shown in parentheses.

Substrate(s)

NADH

Succinate

Citrate + malate

2-Oxoglutarate + malate

Ascorbate + TMPD
State 3 respiration rate [ng-atom $\mathrm{O} \mathrm{min}^{-1}(\mathrm{mg} \text { protein })^{-1}$ ]
Respiratory control ratio

$1 \cdot 16 \pm 0.02$ (3)

$1.38+0.03(12)$

$1.63 \pm 0.17$ (3)

$2.07 \pm 0.04(4)$ none
ADP :O

ratio
$115 \pm 3(12)$
$19.8 \pm 4.8(3)$
$59.7 \pm 3.9(4)$
$179+10(4)$
$0.61 \pm 0.00(3)$

$0.71 \pm 0.04(12)$

$1.51 \pm 0.04$ (3)

$1.42 \pm 0.02(4)$ none

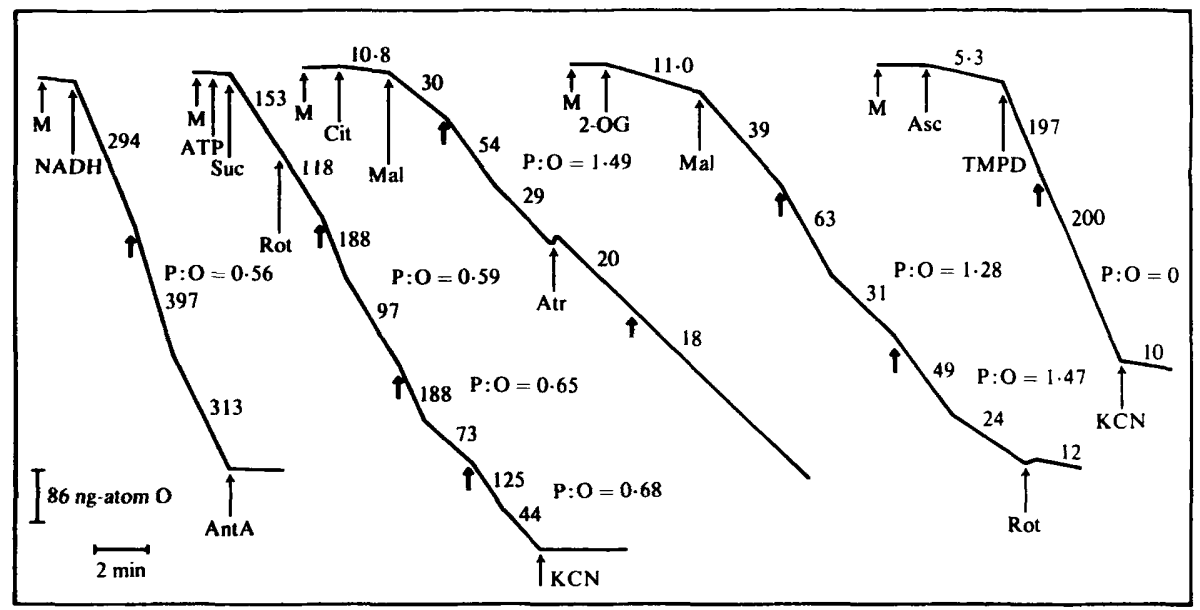

Fig. 1. Oxidation of NADH, succinate (Suc) + $120 \mu \mathrm{M}$-ATP, citrate (Cit) + malate (Mal), 2-oxoglutarate (2-OG) + malate, and ascorbate (Asc) + TMPD by mitochondria (M) isolated from conidia of $N$. crassa. Concentrations of substrates and ADP were as indicated in Table 1. Concentrations of inhibitors were: antimycin A (AntA), 0.3 $\mu \mathrm{M}$; rotenone (Rot), $30 \mu \mathrm{M}$; atractyloside (Atr), $120 \mu \mathrm{M}$; cyanide $(\mathrm{KCN}), 1 \mathrm{~mm}$. The numbers beside each trace show the rate of oxygen uptake in $\mathrm{ng}$-atom $\mathrm{O}$ $\mathrm{min}^{-1}$ (mg protein) $)^{-1}$. ADP was added as indicated by the unlettered arrows. The reactions were carried out at $25^{\circ} \mathrm{C}$ with 0.4 to $0.8 \mathrm{mg}$ protein.

Electron transport in conidial mitochondria was found to be coupled to oxidative phosphorylation (Fig. 1). Coupling of exogenous NADH oxidation to phosphorylation was optimal when the ratio of ADP to mitochondrial protein was $0.24 \mu \mathrm{mol}$ ADP (mg protein) ${ }^{-1}$. Phosphorylation efficiencies (ADP:O ratio) for $\mathrm{NAD}^{+}$-linked substrates were also measured (Table 1). These values were roughly twice as high as those obtained for exogenous NADH and succinate. Nevertheless, the efficiency of energy coupling was less than that reported for mitochondria isolated from mycelia of $N$. crassa (Weiss et al., 1970; Lambowitz et al., $1972 b)$.

\section{Inhibitors of electron transport and oxidative phosphorylation}

Oxidation of all substrates was inhibited 95 to $100 \%$ by the addition of $0.3 \mu \mathrm{M}$-antimycin A (except ascorbate + TMPD) or $1 \mathrm{mM}-\mathrm{KCN}$, which are both known to be effective inhibitors of electron transport, between cytochromes $b$ and $c_{1}$ and at cytochrome $a a_{3}$, respectively. 


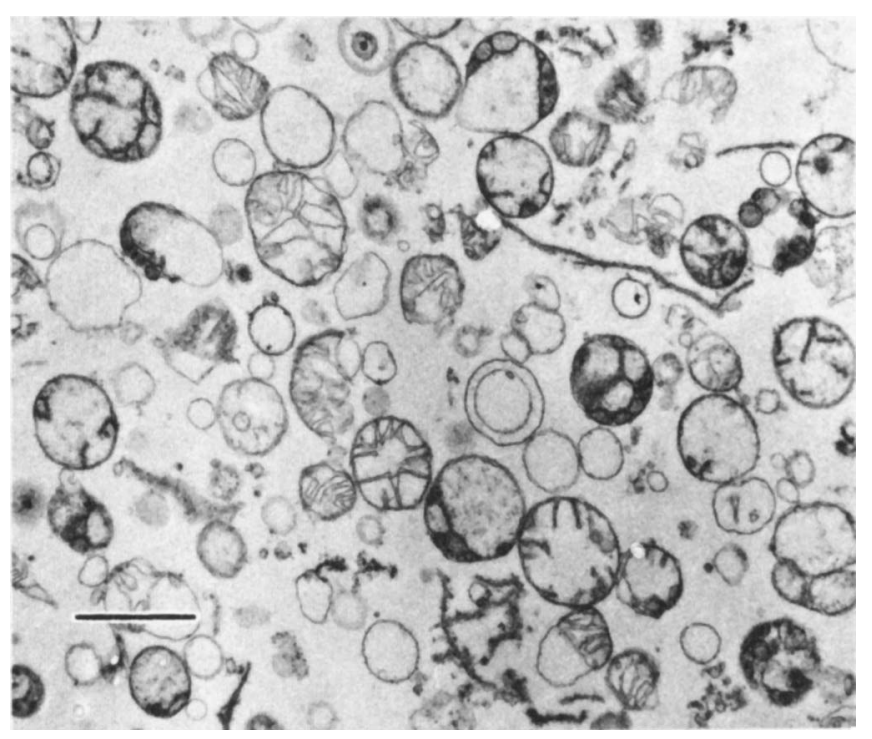

Fig. 2. Electron micrograph of mitochondria isolated from conidia of $N$. crassa. The mitochondrial pellet obtained after centrifugation at $12000 \mathrm{~g}$ was fixed in buffered glutaraldehyde as described in Methods. Mitochondria with double membranes and long or short cristae are visible. The mitochondrial matrices appear clear or finely granular. The bar marker represents $1 \mu \mathrm{m}$.

Rotenone at $30 \mu \mathrm{M}$ inhibited the oxidation of succinate by up to $10 \%$. The inhibition of exogenous NADH oxidation was unexpectedly and for unknown reasons higher $(50 \%)$. Oxidation of $\mathrm{NAD}^{+}$-linked substrates was partially sensitive to the effect of rotenone. Addition of the inhibitor to respiring mitochondria in state 4 decreased oxygen consumption to $30 \%$ of the initial value.

In the presence of $10 \mu \mathrm{g}$ oligomycin, the rate of oxidation for all substrates tested was slightly inhibited (10\%), while, as expected, oxidative phosphorylation was abolished. Inhibition of adenine nucleotide translocase by the addition of $120 \mu \mathrm{M}$-atractyloside (Klingenberg, 1976) had the same effect on oxidative phosphorylation (Fig. 1).

The addition of $60 \mu \mathrm{M}$-2,4-dinitrophenol increased the rate of oxidation of all substrates, except ascorbate + TMPD, by $1 \cdot 3$ - to $1 \cdot 5$-fold and prevented the return to state 4 respiration.

\section{Isopycnic centrifugation}

Two bands of mitochondria were recovered after isopycnic centrifugation, at densities of 1.195 and $1.212 \mathrm{~g} \mathrm{ml}^{-1}$. The former represented the majority of the mitochondrial population. Each band was assayed for its respiratory properties in the presence of succinate and exogenous NADH as substrates. The oxidation rate of succinate was identical in both bands and when statistically compared with the rate in the mitochondrial pellet the difference was not significant. The oxidation rate of exogenous NADH by gradient-purified mitochondria was about $30 \%$ lower than the values obtained with the mitochondrial pellet.

ADP:O and respiratory control ratios were well preserved in both bands and reached the values obtained for the mitochondrial pellet with succinate and NADH. Oxygen consumption of the two bands was totally inhibited by the addition of $0.3 \mu \mathrm{M}$-antimycin A or $1 \mathrm{mM}-\mathrm{KCN}$.

\section{Electron microscopy}

Conidial mitochondria from the $12000 \mathrm{~g}$ pellet appeared to be relatively heterogeneous (Fig. 2). The majority of the mitochondria exhibited double membranes, some of them possessing a reduced number of thin and short cristae within a clear matrix, and others 
showing swollen cristae within a more electron-dense matrix; a few had thin and relatively long cristae. These mitochondria were intermingled with other membranous structures and empty vesicles which were unavoidably produced by the process of mechanical disruption.

\section{DISCUSSION}

Disruption of conidia of $N$. crassa was improved and simplified by the use of a modified Vortex mixer; this led to increased recovery of the mitochondrial fraction compared with previous results (Ortega Perez et al., 1977). Under these new conditions, we obtained a greater proportion of mitochondria with a density of $1.195 \mathrm{~g} \mathrm{ml}^{-1}$, similar to that of mycelial mitochondria (Michéa-Hamzehpour et al., 1979), than with a density of $1.212 \mathrm{~g} \mathrm{ml}^{-1}$, previously considered as characteristic of conidia (Ortega Perez et al., 1977). This mitochondrial heterogeneity might arise directly from the increased recovery and improved preservation of the mitochondria.

The ultrastructural integrity of the isolated mitochondria was confirmed by the similarity of their internal organization to that of conidial mitochondria of $N$. crassa studied in situ (Weiss \& Turian, 1966; Michéa-Hamzehpour et al., 1980). They were also clearly distinguishable from those isolated from mycelia (Michéa-Hamzehpour et al., 1979).

The criteria of integrity of mitochondria isolated by our procedure were in accordance with the general criteria proposed by Lloyd (1979), i.e. negligible endogenous respiration, stimulation of respiratory rate by the addition of ADP (state 3) and subsequent return to state 4 respiration and stimulation of citrate and 2-oxoglutarate oxidation rates by the addition of malate.

In determining respiratory properties of conidial mitochondria, various mitochondrial preparatory media were used. The addition of DTT to the extraction buffer increased both the oxygen uptake rate and the ADP:O ratios at site 1. In fact, without protecting SH groups of enzymes known for their participation in oxidative phosphorylation, metabolite transport (Gautheron, 1973) or other respiratory functions, we were unable to obtain mitochondria that exhibited respiratory control, and the respiratory rate was drastically reduced.

Reducing equivalents from succinate and external NADH, which enter the respiratory chain at the quinone level, did not show such a requirement since, with or without DTT, oxidation of these substrates was associated with energy conservation. Thus site 1 phosphorylation and, as a whole, complex I (NADH-ubiquinone reductase) of the electron transport chain in conidial mitochondria appear labile and care must be taken to preserve their activity during extraction.

We have shown that mitochondria isolated from conidia of $N$. crassa respire via a cyanideand antimycin-sensitive pathway which is partially coupled to oxidative phosphorylation. The highest rate of respiration was obtained with NADH as already found with mycelial mitochondria (Hall \& Greenawalt, 1967; Weiss et al., 1970; Lambowitz et al., 1972 a). Two sites of phosphorylation (sites 1 and 2) were observed to operate during respiration with $\mathrm{NAD}^{+}$-linked substrates, and one site with exogenous NADH or succinate. Site 3 of energy coupling was not observed with ascorbate + TMPD. As a control (not shown), the uptake of inorganic phosphate was measured as described by Coruzzi et al. (1979) in the presence of ADP during oxidation of succinate. A P/O ratio of 0.58 was obtained, which is quite close to the results shown in Table 1.

The functional and coupled mitochondria obtained from ungerminated conidia of $N$. crassa using the method described here were similar to those isolated by Hall \& Greenawalt (1964), except for the fact that our conidial mitochondria could additionally oxidize $\mathrm{NAD}^{+}$-linked substrates. It appears, therefore, that the conidial respiratory system is already active before initiation of germination. This is consistent with the recent report of Brambl (1981) which showed that in Neurospora the respiratory membranes required for conidial germination may be assembled into a functional form before spore dormancy. 
In conclusion, conidial mitochondria of $N$. crassa possess a functional electron transport chain, but with moderate energy conservation as compared with that of mycelial mitochondria isolated by the same treatment (results not shown). The reduced number of cristae and the clear matrix in the conidial mitochondria may perhaps reflect their lower respiratory capacity.

We wish to thank Dr F. Vanderhaeghe for critical reading of the manuscript and Miss E. Herz for technical assistance.

\section{REFERE N C E S}

BRAMBL, R. (1981). Respiration and mitochondrial biogenesis during fungal spore germination. In The Fungal Spore: Morphogenetic Controls, pp. 585604. Edited by G. Turian and H. Hohl. London: Academic Press.

Chance, B. \& Williams, G. R. (1955). A simple and rapid assay of oxidative phosphorylation. Nature, London 175, 1120-1121.

Colvin, H. J., Sauer, B. L. \& Munkres, K. D. (1973). Respiration of wild type and extrachromosomal mutants of Neurospora crassa. Journal of Bacteriology 116, 1314-1321.

Coruzzi, G., Trembath. M. K. \& Tzagoloff. A. (1979). The isolation of mitochondrial and nuclear mutants of Saccharomyces cerevisiae with specific defects in mitochondrial functions. Methods in Enzymology 56, 95-106.

Gautheron. D. C. (1973). Existe-t-il des critères et des propriétés permettant de discriminer entre les différents groupements thiols impliqués dans les translocations mitochondriales et le mécanisme de couplage? Biochimie 55. 727-745.

Greenawalt, J. W., Beck. D. P. \& Hawley, E. S. (1972). Chemical and biochemical changes in mitochondria during morphogenetic development of Neurospora crassa. In Biochemistry and Biophysics of Mitochondrial Membranes, pp. 541-558. Edited by J. F. Azzone \& A. P. Carafoli. New York: Academic Press.

Hall. D. O. \& Greenawalt, J. W. (1964). Oxidative phosphorylation by isolated mitochondria. Biochemical and Biophysical Research Communications 17. 565-569.

Hall. D. O. \& Greenawalt, J. W. (1967). The preparation and biochemical properties of mitochondria from Neurospora crassa. Journal of General Microbiology 48, 419-430.

KLINGENBERG, M. (1976). The ADP-ATP carrier in mitochondrial membranes. In Enzymes of Biological Membranes, vol. 3, pp. 383-438. Edited by A. Martonosi. New York: Plenum Publishing Corp. lambowitz, A. M., Smith, E. W. \& Slayman. C. W.
$(1972 a)$. Electron transport in Neurospora mitochondria. Journal of Biological Chemistry 247, 4850-4858.

Lambowitz, A. M., Smith, E. W. \& Slayman, C. W. $(1972 b)$. Oxidative phosphorylation in Neurospora mitochondria. Journal of Biological Chemistry 247, 4859-4865.

LLOYD, D. (1979). General methodology for isolation and characterization of mitochondria from microorganisms. Methods in Enzymology 55, 135-144.

Michéa-Hamzehpour, M., Ortega Perez, R., Rougemont, A., VANDERHAEghe, F., KHANDJIAN, E., Ton That, T. C. \& Turian, G. (1979). Isolation of two mitochondrial populations with differential hydroxamate-sensitivity from the pokv mutant of Neurospora crassa. FEMS Microbiology Letters 6 , 213-218.

Michéa-Hamzehpour, M., Grange, F., Ton That, T. C. \& Turian, G. (1980). Heat-induced changes in respiratory pathways and mitochondrial structure during microcycle conidiation of Neurospora crassa. Archives of Microbiology 125, 53-58.

Ortega Perez, R. D., Khandjian, E. W., RougeMONT, A., OJHA, M. \& TuRIAN, G. (1977). Changes of mitochondrial density during differentiation of Neurospora crassa. FEMS Microbiology Letters 2, 207-209.

SPURR, A. R. (1969). A low viscosity epoxy resin embedding medium for electron microscopy. Jour nal of Ultrastructural Research 26, 31-43.

voN JAGOW, G. WeISS, H. \& KLINGENBERG, $M$. (1973). Comparison of the respiratory chain of Neurospora crassa wild type and the mi-mutants $\mathrm{mi}-1$ and mi-3. European Journal of Biochemistry 33, 140-157.

WeISS, B. \& TURIAN, G. (1966). A study of conidiation in Neurospora crassa. Journal of General Microbiology 44, 407-418.

Weiss, H., von Jagow, G., KLingenberg, M. \& BüCHER, T. (1970). Characterization of Neurospora crassa mitochondria prepared with a grindmill. European Journal of Biochemistry 14, 75-82. 